Journal of

Cardiology and Vascular Medicine

\title{
Severe Hepatic Toxicity with Portal Hypertension: An Unusual but Preventable Complication of Amiodarone Therapy
}

Shruthi Nammalwar $\mathrm{BS}^{1, *}$, Nima Karamooz $\mathrm{MD}^{2}$, Maggie So $\mathrm{MD}^{2}$

${ }^{1}$ Geisel School of Medicine at Dartmouth, Hanover, NH

${ }^{2}$ Department of Medicine, California Pacific Medical Center, San Francisco, CA

*Corresponding author: Nima Karamooz, Department of Medicine, California Pacific Medical Center, 1101 Van Ness Avenue, Suite 1120 San Francisco, CA 94109, Fax: 415-447-6322

Received Date: May 26, 2019 Accepted Date: June 19, 2019 Published Date: June 20, 2019

Citation: Shruthi Nammalwar (2019) Severe Hepatic Toxicity with Portal Hypertension: An Unusual but Preventable Complication of Amiodarone Therapy. J Cardio Vasc Med 5: 1-5.

\begin{abstract}
A 77 year old African American woman with atrial fibrillation treated with amiodarone 200 mg daily for 67 months presented with new onset ascites, watery diarrhea, and peripancreatic lymphadenopathy. She had a mild elevation of her transaminases and alkaline phosphatase, though initial workup centered on ruling out malignancy. Ultimately, her liver biopsy demonstrated evidence of amiodarone induced hepatotoxicity. She had persistent liver damage requiring frequent paracenteses. This case highlights the importance of reassessing the need for amiodarone and performing routine recommended screening of liver chemistries during treatment.
\end{abstract}

\section{Introduction}

Amiodarone remains one of the most frequently prescribed antiarrhythmics for rhythm control in atrial fibrillation [1,2]. In 2010, the estimated prevalence of atrial fibrillation in the United States ranged from 2.7 - 6.1 million persons with prevalence expected to increase to 12.1 million by 2030 [3].

As the use of amiodarone remains widespread, the myriad side effects affecting the lungs, eyes, skin, thyroid and liver must be considered. Hepatotoxicity occurs as a result of accumulation of amiodarone and its toxic metabolite, $\mathrm{N}$-desethylamiodarone, in the liver which inhibits normal lysosome function, leading to steatohepatitis and potentially cirrhosis [4].
The exact prevalence of amiodarone hepatotoxicity is difficult to assess, however, studies suggest that $25 \%$ of patients may experience asymptomatic transaminitis while 1-3\% of patients may experience symptomatic liver failure [4,5].

The presentation of the latter group can be subtle and varied. Here, we present an unusual case of amiodarone hepatotoxicity that was initially concerning for intra-abdominal malignancy. 
Case

A 77 year old African American woman with a history of latent tuberculosis treated with isoniazid 15 years ago, chronic kidney disease, and paroxysmal atrial fibrillation on $200 \mathrm{mg}$ amiodarone daily for 67 months and rivaroxaban presented to her primary care provider with chief complaint of bloating, dyspnea on exertion, and watery diarrhea. Labs were notable for a mild transaminitis and a predominantly elevated alkaline phosphatase with AST of 109, ALT of 71, and ALP of 385. Labs were otherwise notable for a fasting blood sugar of 71 , a normal serum LDH of 183 , a low serum total protein of 5.8 , and a low serum albumin at 3.1.

Infectious workup was negative. Abdominal ultrasound revealed a normal appearing liver, a $2.0 \times 1.9 \times 2.3 \mathrm{~cm}$ hypoechoic mass in the pancreatic head, and a small volume of ascites. Abdominal MRI confirmed the presence of a mass over the pancreatic body (Figure 1). Endoscopic ultrasound (EUS) surprisingly revealed prominent peripancreatic lymphadenopathy without evidence of pancreatic mass. Biopsies of the lymph nodes demonstrated normal lymphoid tissue.
Over the next three weeks, the patient developed worsening abdominal distention, shortness of breath, lower extremity edema, and diarrhea before presenting to the hospital for further evaluation.

On admission, she continued to have a mild transaminitis with elevated alkaline phosphatase to 434 and elevated gamma-glutamyl transferase to 2,136. Abdominal CT was notable for large volume ascites as well as small granulomas in the liver and spleen stable from prior CT 5 years ago. A paracentesis performed showed a mildly elevated serum-ascites albumin gradient (SAAG) of 1.2 concerning for portal hypertension. Ascitic fluid was notable for 66,500 RBCs with a total protein of 2.5 , albumin of 1.1, and LDH of 75. Her CA-125 tumor marker was mildly elevated at 169.4. Despite high suspicion for malignancy, PET-CT was negative. Transthoracic echocardiogram found no evidence of heart failure or pulmonary hypertension.

A transjugular liver biopsy measured a gradient of $14 \mathrm{mmHg}$ consistent with portal hypertension. Biopsy results showed sclerosing hyaline necrosis without significant steatosis, suggestive of alcoholic liver disease or amiodarone-induced liver injury (Figures $2 \& 3$ ). The patient had no history of alcohol use.

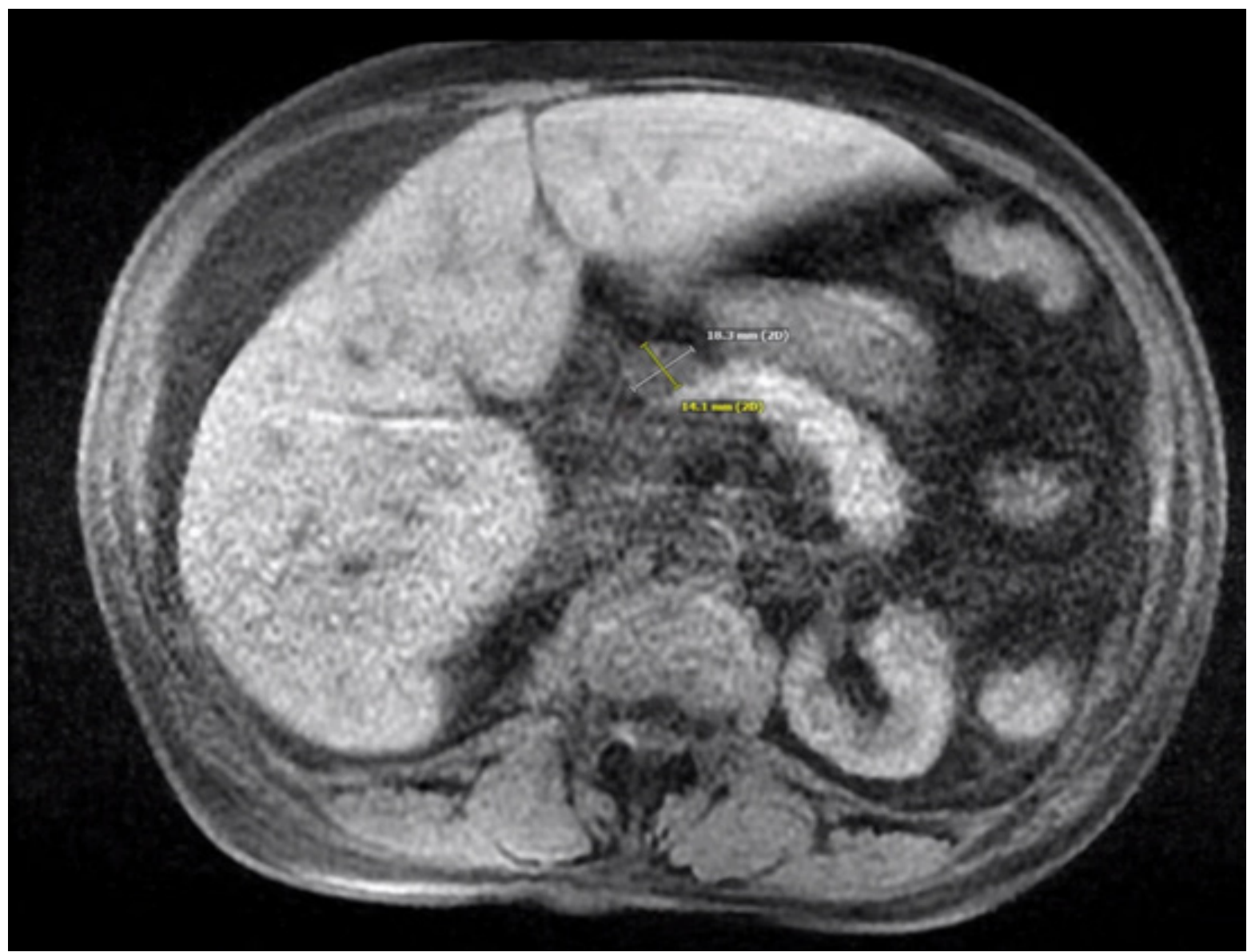

Figure 1: MRI demonstrating normal liver capsule with a $1.8 \times 1.4 \mathrm{~cm}$ mass in the anterior aspect of the pancreatic body. 
Amiodarone was discontinued on that admission, however, 6 months post discharge the patient has continued to require paracenteses every 2 months. Her LFTs at follow up showed a normalized ALT to 19, but with ongoing elevation of AST to 61 and ALP to 248.

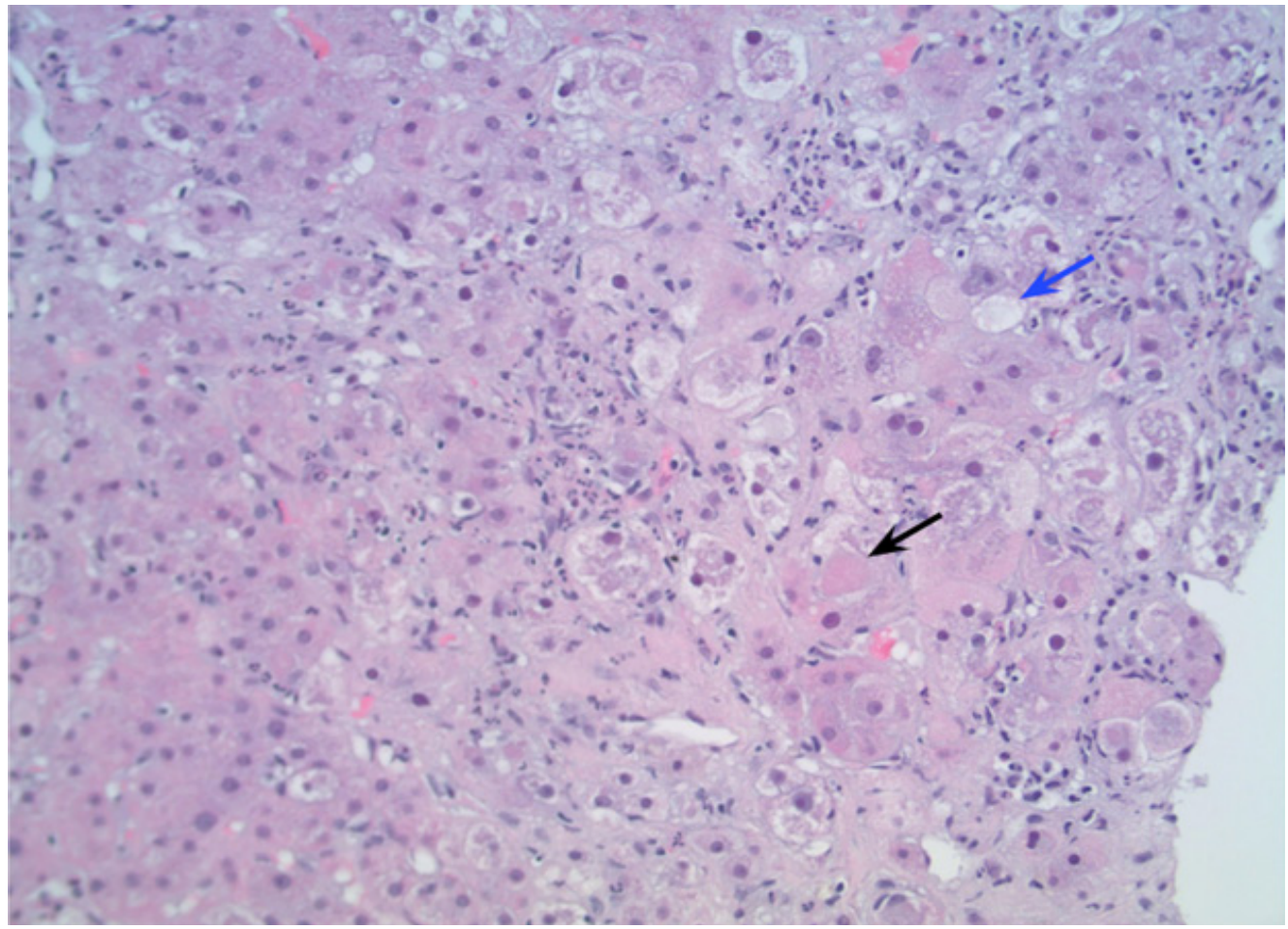

Figure 2: H\&E stain at 200X magnification showing inflammation with Mallory's hyaline (black arrow) with few areas of balloon degeneration reflective of mild steatosis (blue arrow).

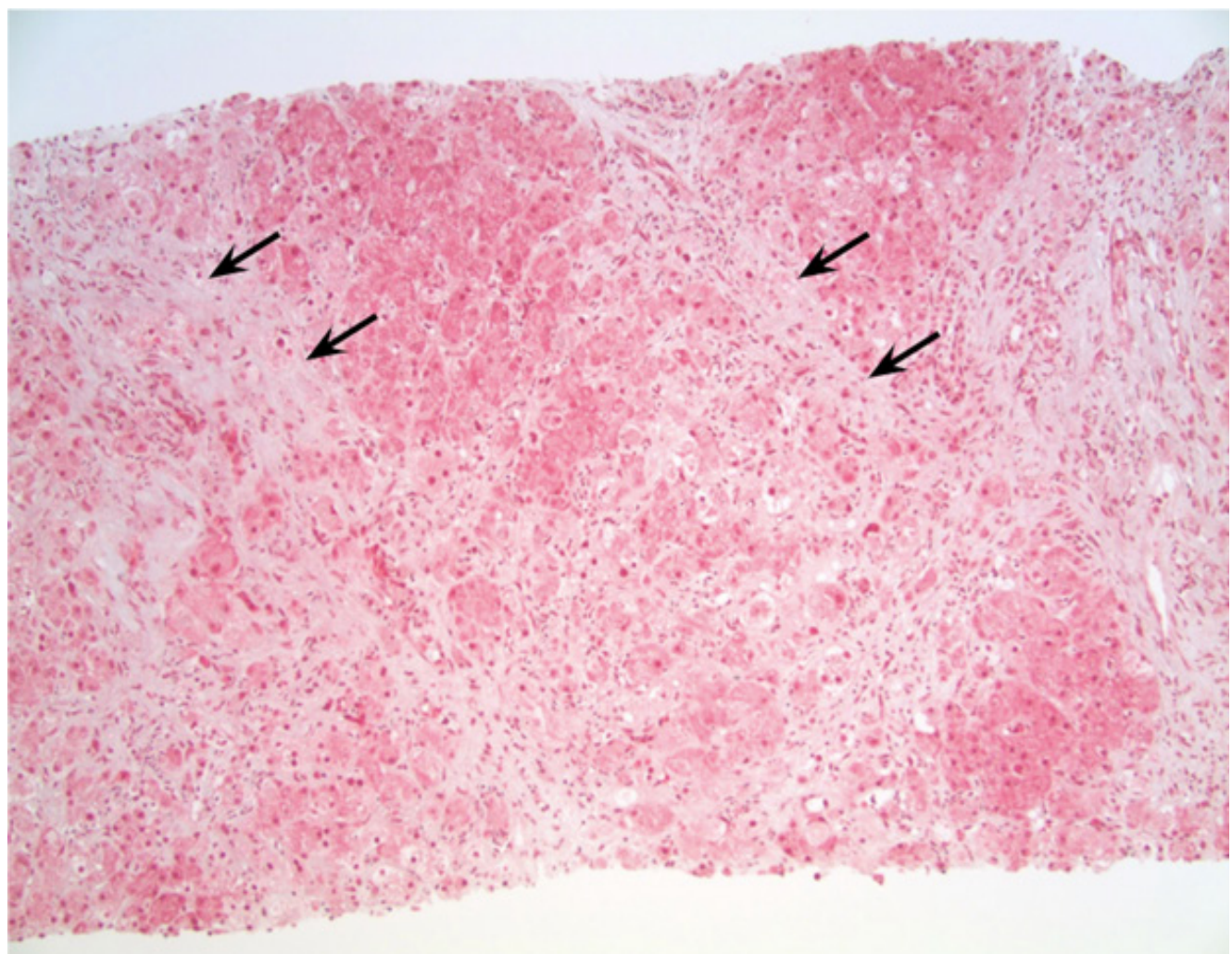

Figure 3: Trichrome stain at 100X magnification demonstrating fine chicken-wire fibrosis (black arrows) without cirrhosis or significant steatosis. 


\section{Discussion}

This report describes an unusual presentation of amiodarone hepatotoxicity in a woman exposed to a total dose of more than 400 grams of amiodarone over a five and a half year period. The initial finding of peripancreatic lymphadenopathy and an elevated CA-125 sparked concern for malignancy before the correct diagnosis was made. Her elevated CA-125 was ultimately attributed to the presence of significant ascites [6]. Total cumulative dose of amiodarone is thought to best correlate with risk of toxicity, and doses as low as 140 grams have been associated with pulmonary toxicity $[7,8]$. The prognosis of patients with hepatotoxicity related to amiodarone is not well documented in the literature. In one review article among patients who progressed to cirrhosis, mortality was $60 \%$ at 5 months following the diagnosis [9]. Previous reports among patients with asymptomatic transamanitis described improvements in liver function tests over a wide time period ranging from 2 weeks to 8 months following discontinuation of the drug $[5,10]$. However, among patients without evidence of cirrhosis on biopsy but with significant ascites, as in our patient, prognostic information has not been documented.

According to Vassallo et al, all patients who are treated with amiodarone should have a TSH and liver enzymes checked every 6 months, along with a yearly chest X-ray to monitor for evidence of pulmonary toxicity [11].

Despite regular follow up visits with her physicians, this patient did not have regular screening with liver enzymes. This case highlights the risk of clinical inertia when starting patients on amiodarone, and emphasizes the importance of regularly reassessing the need for its use, particularly among patients who have a high expected 5-year survival. Furthermore, a best practice advisory could potentially be incorporated in electronic health records to flag patients who are on amiodarone and have not had appropriate surveillance screening.

\section{Acknowledgements}

Richard Garcia-Kennedy for providing histology photos (Figures 2 and 3)

\section{References}

1. Hayward C, Patel HC, Patel K, et al. (2016) The evolving landscape of oral anti-arrhythmic prescriptions for atrial fibrillation in England: 1998-2014. European heart journal Cardiovascular pharmacotherapy 2:90-94.

2. Khan I, Patel HC, Nanayakkara S, Raju H, Voskoboinik A, Mariani JA (2018) Trends in outpatient anti-arrhythmic prescriptions for atrial fibrillation and left atrial ablation in Australia: 1997-2016. Internal medicine journal 48:427-432.

3. Benjamin EJ, Blaha MJ, Chiuve SE, et al. (2017) Heart Disease and Stroke Statistics-2017 Update: A Report From the American Heart Association. Circulation 135:e146-e603.

4. Rabinowich L, Shibolet O (2015) Drug Induced Steatohepatitis: An Uncommon Culprit of a Common Disease. BioMed research international :168905.

5. Lewis JH, Ranard RC, Caruso A, et al. (1989) Amiodarone hepatotoxicity: prevalence and clinicopathologic correlations among 104 patients. Hepatology (Baltimore, Md) 9:679-685.

6. Zuckerman E, Lanir A, Sabo E, et al. (1999) Cancer antigen 125: a sensitive marker of ascites in patients with liver cirrhosis. The American journal of gastroenterology 94:16131618.

7. Brien JF, Jimmo S, Brennan FJ, Ford SE, Armstrong PW (1987) Distribution of amiodarone and its metabolite, desethylamiodarone, in human tissues. Canadian journal of physiology and pharmacology 65:360-364.

8. Dusman RE, Stanton MS, Miles WM, et al. (1990) Clinical features of amiodarone-induced pulmonary toxicity. Circulation 82:51-59.

9. Hussain N, Bhattacharyya A, Prueksaritanond S (2013) Amiodarone-induced cirrhosis of liver: what predicts mortality? ISRN cardiology 617943.

10. Tsuda T, Tada H, Tanaka Y, et al. (2018) Amiodaroneinduced reversible and irreversible hepatotoxicity: two case reports. Journal of medical case reports 12:95. 
11. Vassallo P, Trohman RG (2007) Prescribing amiodarone: an evidence-based review of clinical indications.

Jama 298:1312-1322.

Submit your manuscript to a JScholar journal and benefit from:

ฯ Convenient online submission

- Rigorous peer review

I Immediate publication on acceptance

ๆ Open access: articles freely available online

9. High visibility within the field

ब Better discount for your subsequent articles

Submit your manuscript at

http://www.jscholaronline.org/submit-manuscript.php 